\title{
T2-signal intensity, SSTR expression, and somatostatin analogs efficacy predict response to pasireotide in acromegaly
}

\author{
Eva C Coopmans' , Joppe J Schneiders², Nour El-Sayed', Nicole S Erler³, Leo J Hofland', Aart-Jan van der Lely', \\ Patrick Petrossians ${ }^{4}$, Julia Potorac ${ }^{4}$, Ammar Muhammad ${ }^{1}$ and \\ Sebastian J C M M Neggers' \\ ${ }^{1}$ Department of Medicine, Endocrinology Section, Pituitary Center Rotterdam, The Netherlands, ${ }^{2}$ Department of \\ Radiology, Erasmus University Medical Center, Rotterdam, The Netherlands, ${ }^{3}$ Department of Biostatistics, Erasmus \\ University Medical Center, Rotterdam, The Netherlands, and ${ }^{4}$ Department of Endocrinology, Centre Hospitalier \\ Universitaire de Liège, Domaine Universitaire du Sart Tilman, Liège, Belgium \\ Correspondence \\ should be addressed \\ to E C Coopmans \\ Email \\ e.coopmans@erasmusmc.nl
}

\begin{abstract}
Objective: T2-signal intensity and somatostatin (SST) receptor expression are recognized predictors of therapy response in acromegaly. We investigated the relationship between these predictors and the hormonal and tumoral responses to long-acting pasireotide (PAS-LAR) therapy, which were also compared with responsiveness to firstgeneration somatostatin receptor ligands (SRLS).

Design: The PAPE study is a cohort study.

Methods: We included 45 acromegaly patients initially receiving SRLs, followed by combination therapy with pegvisomant, and finally PAS-LAR. We assessed tumor volume reduction ( $\geq 25 \%$ from baseline), IGF-1 levels (expressed as the upper limit of normal), and T2-weighted MRI signal and SST receptor expression of the adenoma.

Results: Patients with significant tumor shrinkage during PAS-LAR showed higher IGF-1 levels during PAS-LAR (mean (S.D.): $1.36(0.53)$ vs $0.93(0.43), P=0.020$ ), less IGF-1 reduction after first-generation SRLs (mean (S.D.): 0.55 (0.71) vs $1.25(1.07), P=0.028$ ), and lower $\mathrm{SST}_{2}$ receptor expression (median (IQR): $2.0(1.0-6.0)$ vs $\left.12.0(7.5-12.0), P=0.040\right)$. Overall, T2-signal intensity ratio was increased compared with baseline (mean (S.D.): 1.39 (0.56) vs 1.25 (0.52), $P=0.017)$ and a higher T2-signal was associated with lower IGF-1 levels during PAS-LAR $(\beta:-0.29,95 \% \mathrm{Cl}:-0.56$ to $-0.01, P=0.045)$. A subset of PAS-LAR treated patients with increased T2-signal intensity achieved greater reduction of IGF-1 (mean (S.D.): $0.80(0.60)$ vs $0.45(0.39), P=0.016)$.

Conclusions: Patients unresponsive to SRLs with a lower $\mathrm{SST}_{2}$ receptor expression are more prone to achieve tumor shrinkage during PAS-LAR. Surprisingly, tumor shrinkage is not accompanied by a biochemical response, which is accompanied with a higher T2-signal intensity.
\end{abstract}

\section{Introduction}

Acromegaly is a rare endocrine disease characterized by growth hormone (GH) hypersecretion and elevated insulin-like growth factor 1 (IGF-1) levels, generally as a result of a GH-producing pituitary tumor (1). Treatment modalities are aimed at normalizing IGF-1 levels, reducing GH levels below $1.0 \mu \mathrm{g} / \mathrm{L}$, decreasing tumor volume, and improving clinical symptoms (2). Transsphenoidal surgery is considered the gold standard in acromegaly management (2), but medical therapy has an increasingly important role. Pasireotide long-
Published by Bioscientifica Ltd. 
acting release (PAS-LAR) is a somatostatin multi-receptor ligand approved for second-line medical therapy for patients with acromegaly for whom surgery is not an option or is not curative $(3,4)$.

Previous clinical studies have indicated that PAS-LAR can achieve control of GH and IGF-1 levels in a subset of acromegaly patients not responding adequately to first-generation somatostatin receptor ligands (SRLs) and might reduce tumor size in patients uncontrolled with first-generation SRLs $(5,6)$. The PAPE study $(7,8)$ investigated acromegaly patients well-controlled on a combination treatment of first-generation SRLs and the GH receptor antagonist pegvisomant (PEGV). Switching to PAS-LAR with or without PEGV resulted in control of IGF-1 levels in most (77.0\%) patients.

T2-weighted MRI signal of the adenoma has been recently recognized as a non-invasive predictor of response to first-generation SRL therapy in acromegaly. Somatotroph adenomas with lower T2-signal intensity are frequently smaller and less invasive than adenomas with a higher T2-signal intensity $(9,10,11,12)$, but the correlation with first-generation SRL-induced tumor shrinkage was inconsistent among studies (9, 11, 13, 14). Although T2-hypointense adenomas are associated with higher GH levels at diagnosis (10), these patients have greater $\mathrm{GH}$ and IGF-1 reductions after a median of 6 months of presurgical first-generation SRLs treatment than patients with T2-iso- or T2-hyper-intense adenomas $(9,11,14)$.

There is evidence that biochemical response to somatostatin analogs can be predicted by the SST receptor subtype binding profile in the somatotroph adenoma (15, 16). The first-generation SRLs show a high preferential binding affinity for $\mathrm{SST}_{2}$ receptor, while PAS-LAR exhibits particularly high affinity for $\mathrm{SST}_{5}$ receptor (17). The in vitro anti-proliferative (18) and anti-secretory (19) effects of firstgeneration SRLs and PAS-LAR therapy were superimposable. Although the latter suggests a predominant role for the $\mathrm{SST}_{2}$ receptor in mediating the inhibitory effect of PAS-LAR on GH secretion in adenomas, results of the same in vitro study (19) indicated that somatotroph adenomas with lower $\mathrm{SST}_{2}$ receptor expression were better responders to PASLAR compared to first-generation SRLs. To our knowledge, there are no studies investigating the correlation between anti-proliferative effects of PAS-LAR and the SST receptor profile in acromegaly patients.

The aims of this prospective open-label conversion study were: (1.) to investigate the relationship between the T2-weighted signal of the adenoma and the hormonal and tumoral responses to PAS-LAR, alone or in combination with PEGV therapy, including the $\mathrm{SST}_{2}$ and $\mathrm{SST}_{5}$ receptor expression in adenomas and (2.) to investigate to what extent this correlates with responsiveness to firstgeneration SRLs.

\section{Subjects and methods}

\section{Cohort description}

Data collection of acromegaly patients was performed at the outpatient clinic of the Pituitary Center Rotterdam, Erasmus University Medical Center in Rotterdam. We initially started with a cohort of 61 acromegaly patients who received PAS-LAR treatment during their participation in the PAPE study (Fig. 1); details of the study design have been reported $(7,8)$. Briefly, all included patients have previously been treated with first-generation SRLs, followed by PEGV and SRL combination therapy. At baseline, the PEGV dose was reduced by $50 \%$ up to 3 months, while first-generation SRLs were continued. When IGF-1 remained $\leq 1.2 \times$ ULN after 3 months, patients were switched to PAS-LAR $60 \mathrm{mg}$ monotherapy for 3 months. When IGF- 1 was $>1.2 \times$ ULN, patients were switched to PAS-LAR $60 \mathrm{mg}$, and they continued with the 50\% reduced PEGV dose for 3 months. During the extension phase, up to 9 months of PAS-LAR treatment, the goal was to achieve IGF-1 normalization (IGF- $1 \leq 1.2 \times$ ULN) through protocol-based dose titration of PEGV (7).

We prospectively collected data on PAS-LAR, either as monotherapy or in combination with PEGV, while data on medical history and clinical response in patients during first-generation SRLs treatment were collected retrospectively. The PAPE study was registered with ClinicalTrails.gov, number NCT02668172. The study was approved by the independent Medical Ethics Committee of the Erasmus University Medical Center in Rotterdam, and the appropriate data use agreements were in place for the database. Written informed consent was obtained from all study participants prior to inclusion.

Patients who received postoperative radiotherapy $(n=7)$, patients with low quality baseline or follow-up MRI during PAS-LAR, alone or in combination with PEGV $(n=3)$, and patients with an MRI without a visible solid component $(n=6)$ were excluded. In total, 45 patients remained and were finally included in this study cohort. Seventeen out of these 45 patients underwent neurosurgery before the PAPE study.

Immunohistochemistry (IHC) was assessed in patients with sufficient adenoma tissue available $(n=13)$. One patient underwent a second surgery, but we analyzed 


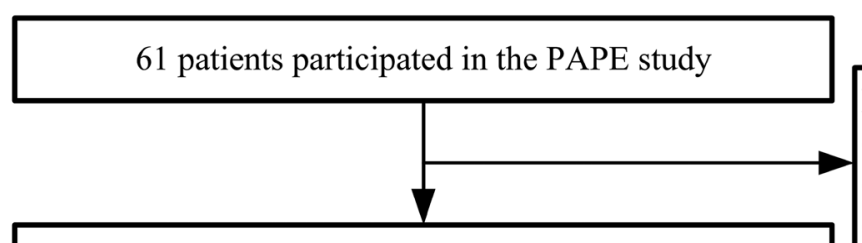

Excluded, $\mathrm{n}=16$

- Previous radiotherapy, $\mathrm{n}=7$

Baseline or follow-up MRI low quality, $\mathrm{n}=3$

MRI without visible solid component, $n=6$

45 patients included in study cohort

17 out of 45 patients received surgery

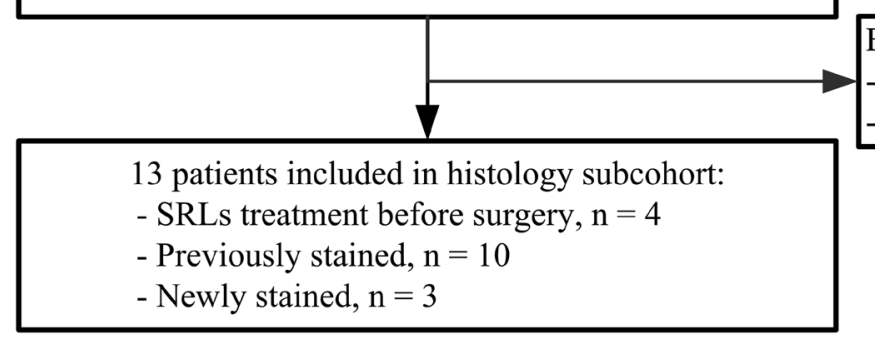

Excluded, $n=4$

- Tissue not available, $\mathrm{n}=2$

- Tissue not sufficient for IHC, $n=2$

\section{Figure 1}

Flowchart of the selection procedure for the study cohort and the somatotroph adenoma tissue samples. All patients eventually received first-generation SRL and PEGV combination treatment and were switched to pasireotide LAR treatment alone or in combination with PEGV during the PAPE study. IHC, immunohistochemistry; MRI, magnetic resonance imaging; PAPE, pasireotide LAR and pegvisomant study; SRL, first-generation somatostatin receptor ligand; SST, somatostatin.

only the tissue sample of the first surgery. From the 13 remaining tissue samples included for IHC analysis, ten tissue samples were stained previously and three were newly stained (Fig. 1).

\section{Biochemical measurements}

Random GH serum concentrations were analyzed using the IDS-iSYS assay (IDS-iSYS, Immunodiagnostic Systems Limited, Boldon, UK), which is free of interference from PEGV (20). Total IGF-1 serum concentrations during the PAPE study were analyzed by the commercially available immunometric assay (IDS-iSYS, Immunodiagnostic Systems Limited, Boldon, UK). Interassay coefficients of variation (CVs) for GH and IGF-1 were $<5 \%$ (GH; $n=190$ ) and (IGF-1; $n=190)$ in serum based internal quality control measurements over a period of 1 year. Total IGF-1 serum concentrations before and after first-generation SRL treatment were analyzed using different assays: Immulite 2000 assay, a solid-phase, validated enzyme-labelled chemiluminescent immunometric assay (DPC Biermann $\mathrm{GmbH} /$ Siemens; intra-assay variability of $2-5 \%$, interassay variability of 3-7\%), the immunometric IDS-iSYS assay (IDSiSYS, Boldon, UK; intra-assay CV: 8.1\%, interassay CV: $2.1 \%$ ), and two different radioimmunoassays (Diagnostic Systems Laboratories, Webster, Tex., USA, intra-assay CV: 3.9\%, interassay CV: 4.2\%, and Medgenix Diagnostics, Fleurus, Belgium; intra-assay CV: 6.1\%, interassay: CV 9.9\%).

IGF-1 concentrations are expressed as the upper limit of normal (ULN; measured IGF-1 divided by the age- and sex-specific ULN) (21).

Response to PAS-LAR, alone or in combination with PEGV, during the PAPE study was divided into short- and long-term treatment response. Short-term treatment response was evaluated as IGF-1 levels ( $\mathrm{x}$ ULN) and as absolute IGF-1 reduction during 3 months of PAS-LAR treatment (i.e. after three injections of PAS-LAR $60 \mathrm{mg}$ ). Absolute IGF-1 reduction was calculated by subtracting IGF-1 ( $x$ ULN) level at baseline (i.e. the start of the study) from IGF-1 (x ULN) level at follow-up. During the extension phase, until 9 months, the PEGV dose was titrated according to a protocol (7) to achieve normalized IGF-1 levels. Therefore, the long-term treatment response to PAS-LAR was based on a composite 'PAS-LAR treatment response score' (PAS-LAR score) in order to fully capture the effect of PAS-LAR, taking into account PEGV dose reduction, discontinuation, and finally PAS-LAR dose reduction (8). The PAS-LAR score comprised five categories (0-4), representing the difference in PEGV dose and PASLAR dose during 9 months of treatment vs baseline (i.e. the start of the study). The highest PAS-LAR score reflects the best response to PAS-LAR treatment. 


\section{MRI evaluation}

Gadolinium-enhanced pituitary MRI was performed at baseline (i.e. the start of the study), or maximum 1 year before study entry, and during 9 months of PAS-LAR, either as monotherapy or in combination with PEGV. The MRI examinations of each patient were collected for centralized, blinded reading. A single experienced neuroradiologist (J S) evaluated the MRIs in the Picture Archive Communication System (PACS, Sectra®, Linköping, Sweden). According to previous literature, the T2-weighted signal on the baseline MRI was visually compared to the normal pituitary tissue and, if not visible, to the gray matter of the temporal lobe $(9,10)$. Moreover, quantification of the T2-weighted MRI signal by Region Of Interest (ROI) measurement of the adenoma, normal pituitary tissue, and gray matter of the temporal lobe was performed and used as ROI-derived T2 intensity ratio of the adenoma vs gray matter.

Tumor volume was calculated according to the formula: height $\times$ width $\times$ length $\times \pi / 6$ (22). Relative tumor shrinkage was calculated by subtracting the tumor volume at baseline from the follow-up measurement, divided by the tumor volume at baseline: a tumor volume change of $\geq 25 \%$ from baseline was considered significant. Absolute tumor shrinkage was calculated by subtracting tumor volume at baseline from tumor volume at follow-up.

Reviewing the tumoral response to first-generation SRLs, diagnostic MRIs as well as follow-up MRIs performed at least after 3 months of first-generation SRL treatment were assessed. In our cohort, we were unable to assess T2-signal intensity of these diagnostic MRIs visually or by ROI measurement due to the overall low resolution. For evaluation of tumor response to first-generation SRL treatment, tumor shrinkage of $\geq 25 \%$ was considered significant.

\section{Immunohistochemistry}

Somatotroph adenoma tissues were stained for hematoxylin and immunostained for $\mathrm{SST}_{2}$ and $\mathrm{SST}_{5}$ receptor as described previously (23). Immunoreactivity of the adenomas for $\mathrm{SST}_{2}$ and $\mathrm{SST}_{5}$ receptor was scored semi-quantitatively using a validated immunoreactivity scoring system (IRS) (24). Two independent investigators (E C and A M) - blinded to each other's findings and the patient's characteristics - scored the protein expression of the newly stained adenoma tissue samples taking into consideration the histopathological description of the sample provided by the pathologist.

\section{Outcomes}

The primary endpoints of the study were tumor volume reduction of $\geq 25 \%$ from baseline and the prediction of the T2-weighted MRI signal for biochemical response and tumor volume reduction during PAS-LAR, alone or in combination with PEGV therapy. Additional endpoints were (1.) SST receptor IHC scores and (2.) absolute IGF-1 reduction achieved after at least 4 months of high dose first-generation SRL treatment. The biochemical response to PAS-LAR, either as monotherapy or in combination with PEGV, was divided into short-term (until 3 months) and long-term (until 9 months) response. The secondary endpoints were absolute tumor shrinkage and random GH serum concentrations.

\section{Statistical analysis}

Continuous data were expressed as mean and S.D. or median and interquartile range (IQR), as appropriate. Categorical data were represented as observed frequencies and percentages. The empirical distributions of continuous variables were plotted and assessed for normality using the Kolmogorov-Smirnov test. Natural logarithmic transformation of the data was done when required. We compared categorical variables between two or more groups with the $\chi^{2}$ test, and we compared continuous variables between groups with either Student's $t$-test or Mann-Whitney $U$ test (for two groups, as appropriate). We tested correlations between continuous variables with either Pearson's $(r)$ or Spearman's rank correlation coefficient $\left(r_{s}\right)$, as appropriate. A multivariable linear regression model was used to investigate associations between candidate predictors (e.g. age, sex, T2-signal intensity ratio, and tumor shrinkage) for the outcome hormonal response during PAS-LAR treatment. Statistical analyses were performed with $\mathrm{R}$ statistical software, version 3.4.1 (packages $r m s$ ), and graphs were drawn using GraphPad Prism version 6 for Windows (GraphPad Software). All tests were two-sided and $\alpha$ was set at 0.05 without any multiplicity correction.

\section{Results}

\section{Cohort demographics and clinical characteristics}

Cohort demographics and clinical characteristics of the 45 included patients receiving PAS-LAR treatment are summarized in Table 1. After 3 months, 10 (22.2\%) out of 
Table 1 Cohort demographics and clinical characteristics of the total group, non-hyperintense group, and hyperintense adenomas subgroup. Data are reported as mean (S.D.), median (IQR), or number (\%).

\begin{tabular}{|c|c|c|c|}
\hline & All patients & $\begin{array}{l}\text { Non-increased T2-signal } \\
\text { intensity subgroup }\end{array}$ & $\begin{array}{l}\text { Increased T2-signal intensity } \\
\text { subgroup }\end{array}$ \\
\hline & $n=45$ & $n=33$ & $n=12$ \\
\hline Age (years) & $52.0(11.7)$ & $51.7(11.8)$ & $52.8(11.9)$ \\
\hline Female patients & $19(42.2 \%)$ & $15(45.5 \%)$ & $4(33.3 \%)$ \\
\hline Previous surgery & $17(37.8 \%)$ & $15(45.5 \%)$ & $2(16.7 \%)$ \\
\hline SRL treatment duration (months) ${ }^{\dagger}$ & $9.9(10.5)$ & $7.9(6.2-10.1)$ & $6.5(4.5-7.9)$ \\
\hline IGF-1 after SRL therapy (x ULN) & $2.22(1.06)$ & $2.20(1.08)$ & $2.29(1.07)$ \\
\hline IGF-1 absolute reduction after SRL therapy (x ULN) & $1.01(1.01)$ & $1.03(0.97)$ & $0.95(1.16)$ \\
\hline PEGV dose at baseline (mg/week) & $80(60-160)$ & $80(20-120)$ & $100(63-175)$ \\
\hline IGF-1 during PAS-LAR 3 months (x ULN) & $1.12(0.49)$ & $1.17(0.51)$ & $0.97(0.43)$ \\
\hline $\begin{array}{l}\text { IGF-1 absolute reduction during PAS-LAR } 3 \text { months } \\
\qquad(x \text { ULN) }\end{array}$ & $0.50(0.52)$ & $0.45(0.39)$ & $0.80(0.60)^{*}$ \\
\hline PAS-LAR score 9 months & $1.5(1.1)$ & $1.5(1.2)$ & $1.5(1.1)$ \\
\hline Macroadenomas & $36(80.0 \%)$ & $24(72.7 \%)$ & $12(100.0 \%)$ \\
\hline Tumor volume $\left(\mathrm{mm}^{3}\right)$ & 1608 (439-4504) & $779(333-3041)$ & $3687(1919-7178)^{*}$ \\
\hline T2-signal adenoma/pituitary or GM at baseline & $1.24(0.50)$ & $1.26(0.52)$ & $1.19(0.47)$ \\
\hline Mean time between MRIs (months) & $18.8(7.3)$ & $19.0(7.8)$ & $18.1(6.0)$ \\
\hline $\mathrm{SST}_{2}$ receptor ${ }^{\dagger \dagger}$ & $9.0(4.0-12.0)$ & $9.0(6.0-12.0)$ & $6.5(1.0-12.0)$ \\
\hline $\mathrm{SST}_{5}$ receptor $^{\mathrm{tt \dagger}}$ & $12.0(7.0-12.0)$ & $12.0(8.0-12.0)$ & $9.0(6.0-12.0)$ \\
\hline $\mathrm{SST}_{2} / \mathrm{SST}_{5}$ receptor ratio ${ }^{\dagger+\dagger}$ & $1.0(0.5-1.4)$ & $1.0(0.6-1.1)$ & $1.0(0.1-2.0)$ \\
\hline
\end{tabular}

Data are reported as mean (s.D.), median (IQR), or number (\%). Asterisk represents $P=\leq 0.05$ for the comparisons between non-hyperintense and hyperintense adenoma group and are derived from the Student's $t$-test (continuous variables) and Pearson's $\chi^{2}$ test (categorical variables).

${ }^{\dagger}$ One patient received first-generation SRL treatment less than 4 months; ${ }^{\dagger \dagger}$ Obtained from 13 tissue samples; ${ }^{\dagger \dagger}$ Obtained from 12 tissue samples. GM, gray matter; IGF-1, insulin-like growth factor 1; PAS-LAR, pasireotide long-acting release; PEGV, pegvisomant; SRL, first-generation somatostatin receptor ligand; ULN, upper limit of normal.

45 patients were on PAS-LAR $60 \mathrm{mg}$ monotherapy every 4 weeks, increasing to 31 (68.9\%) patients during 9 months of PAS-LAR treatment. The remaining 14 (31.1\%) patients with elevated IGF-1 levels continued with their reduced dose of PEGV treatment in combination with PAS-LAR $60 \mathrm{mg}$ monthly. In this combination group, the average total reduction in PEGV dose was 32.9\% (median $30 \mathrm{mg} /$ week (IQR 0-95) vs $80 \mathrm{mg} /$ week (60-160), $P \leq 0.0001)$ in combination with PAS-LAR after 9 months of treatment.

We confirmed that quantitative measurement defined as ROI-derived T2 intensity ratio of the adenoma vs pituitary tissue on MRI corresponded well with the visual assessment at baseline. At the end of the study, the mean T2-signal intensity ratio of the adenoma in the total cohort during PAS-LAR treatment was significantly higher compared with baseline (mean (S.D.): 1.39 (0.56) vs 1.25 (0.52), $P=0.017)$.

\section{Tumoral response}

Tumoral response was evaluated after a mean of 11.8 months of PAS-LAR treatment. In 33 patients (73.3\%), tumor volume decreased from baseline; this shrinkage was significant ( $\geq 25 \%$ ) in only 15 cases (33.3\%) (Fig. 2). Tumor volume increased from baseline in 12 patients $(26.7 \%)$, of which $6(13.3 \%)$ had a significant $(\geq 25 \%)$ tumor volume increase. Three patients harbored microadenomas without clinically relevant tumor size increase. The other three harbored macroadenomas and the observed significant increase in tumor size was only clinically relevant in one patient (e.g. surgical intervention and/or stereotactic radiosurgery is needed). With regard to the

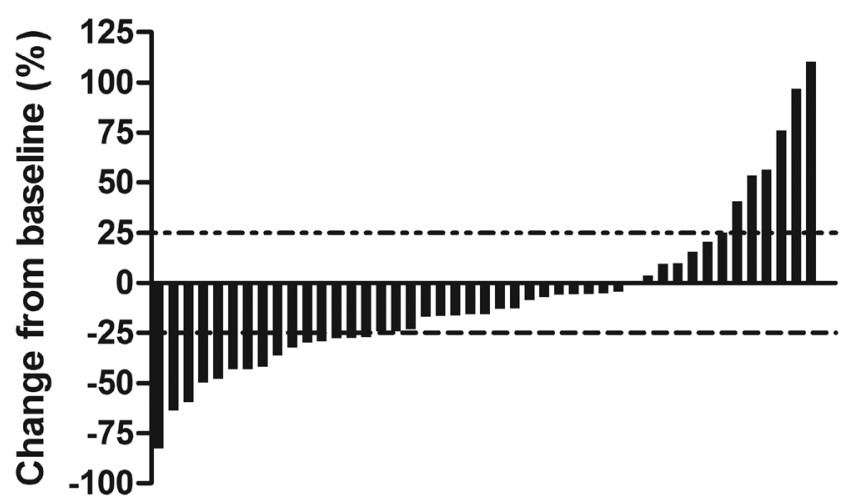

Figure 2

Change in tumor volume from baseline to follow-up (\%). The dashed line represents $25 \%$ reduction for tumor volume. Figure shows relative change for tumor volume in individual patients with available data at baseline and follow-up. 
tumoral response to first-generation SRL treatment, tumor shrinkage was significant ( $\geq 25 \%)$ in 13 cases $(28.9 \%)$. In a previous study, our group observed in approximately the same cohort a significant tumor shrinkage $(\geq 20 \%)$ in 13 (16.9\%) out of 77 patients during combination treatment with first-generation SRLs and PEGV (25). Interestingly, an additional significant decrease in tumor volume was observed during PAS-LAR in two cases (15.4\%).

At baseline, tumor size was correlated with random GH levels $(r=0.85, P \leq 0.0001)$. Moreover, larger adenomas correlated with greater absolute tumor shrinkage during PAS-LAR treatment $(r=0.51, P=0.00038)$. Microadenomas tended to present more often with higher T2-signal intensity at baseline, compared with macroadenomas (mean (S.D.): $1.58(0.60)$ vs $1.18(0.47), P=0.06)$. T2-signal intensity at baseline had no effect on significant tumor shrinkage (mean (S.D.): 1.12 (0.35) vs 1.31 (0.57), $P=0.23$ ).

With regard to the hormonal responsiveness, patients with significant tumor shrinkage during PAS-LAR treatment had less IGF-1 reduction after first-generation SRL treatment when compared to the total cohort (mean (S.D.) x ULN: 0.55(s.D. 0.71) vs 1.25 (1.07), $P=0.028$; Fig. 3A). After 3 months of PAS-LAR treatment, patients with significant tumor shrinkage had higher IGF-1 levels than patients without significant tumor shrinkage (mean (S.D.) x ULN: 1.36 (0.53) vs 0.93 (0.43), $P=0.020$; Fig. 3B).

\section{T2-signal intensity of the adenoma}

The visual assessment of T2-weighted MRI signal categorized $12(26.7 \%)$ adenomas as hypointense, 13 (28.9\%) as isointense, and $20(44.4 \%)$ as hyperintense at baseline.

We observed an inverse correlation between IGF-1 ( $\mathrm{x}$ ULN) levels during 3 months of PAS-LAR treatment and T2-signal intensity of adenomas at baseline $(r=-0.39$, $P=0.0075$; Fig. 4A). In other words, higher T2-signal intensity adenomas at baseline are correlated with better hormonal response (i.e. meaning lower IGF-1 (x ULN) levels during 3 and 9 months of PAS-LAR treatment).

\section{Increased T2-signal intensity of the adenoma}

As recently reported, in 14 patients T2-weighted MRI signal intensity of the adenoma was increased during PASLAR treatment (26). Two out of 14 patients with increased T2-weighted MRI signal intensity of the adenoma received
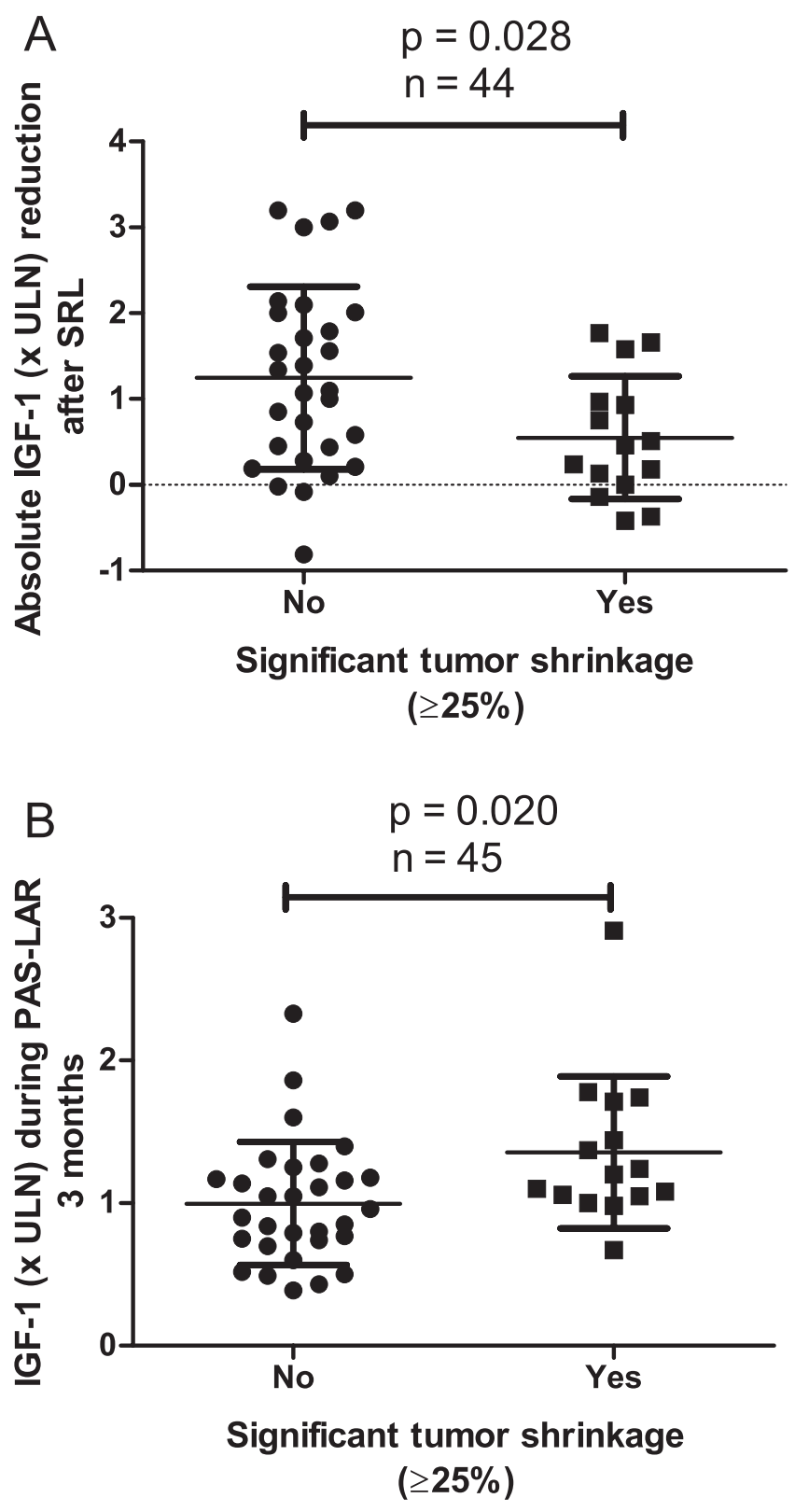

Figure 3

Relation between significant tumor shrinkage and hormonal response to SRL treatment and PAS-LAR treatment. Data are presented as scatter dot plots depicting the mean (s.D.). (A) The absolute IGF-1 (x ULN) reduction after SRL treatment was greater in patients without significant tumor shrinkage, and $(B)$ the IGF-1 ( $x$ ULN) levels during 3 months of PAS-LAR are lower in patients without significant tumor shrinkage. IGF-1, insulin-like growth factor 1; PAS-LAR, pasireotide long-acting release; SRL, first-generation somatostatin receptor ligand; ULN, upper limit of normal. 


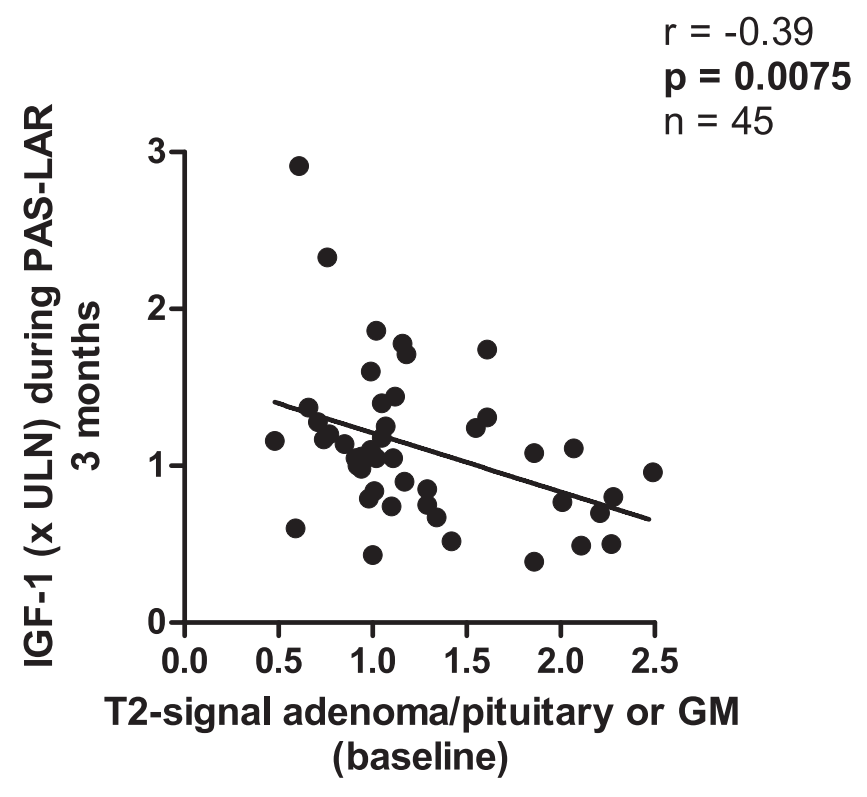

Figure 4

Relation between T2-weighted MRI signal intensity of the adenomas and hormonal response to PAS-LAR treatment. Data is presented as a scatter plot with Pearson correlation coefficient ( $r$ ). (A) T2-signal intensity adenomas at baseline were inversely correlated to IGF-1 (x ULN) levels during 3 months of PAS-LAR treatment. GM, gray matter; IGF-1, insulin-like growth factor 1 ; PAS-LAR, pasireotide long-acting release; ULN, upper limit of normal.

postoperative radiotherapy and were excluded from the following analysis.

At baseline, we did not observe any significant differences in age, sex, and biochemical response after first-generation SRL treatment or T2-signal intensity ratio between patients with increased T2-signal intensity and patients with non-increased T2-signal intensity (Table 1). Patients with increased T2-signal intensity had larger adenomas at baseline compared to the total cohort (median (IQR): $2687 \mathrm{~mm}^{3}$ ( 1919-7178) vs $779 \mathrm{~mm}^{3}$ (333-3041), $P=0.026$; Table 1). Furthermore, we found no significant differences in absolute IGF-1 reduction after first-generation SRL treatment in patients with increased T2-signal intensity adenomas (mean (S.D.) x ULN: 0.95 (1.16) vs 1.03 (0.97); $P=0.83$; Table 1 ). These patients further showed greater reduction of IGF-1 levels during 3 months of PAS-LAR treatment (mean (S.D.) x ULN: 0.80 (0.60) vs 0.45 (0.39), $P=0.016$; Table 1$)$. However, they did not present more often with significant tumor shrinkage (4 patients (33.3\%) vs 11 patients (33.3), $\chi^{2} P=1.00$ ) nor with greater absolute tumor shrinkage during PAS-LAR (median (IQR): $146 \mathrm{~mm}^{3}$ (-25to1199) vs $110 \mathrm{~mm}^{3}$ (5-449); $P=0.79$ ).
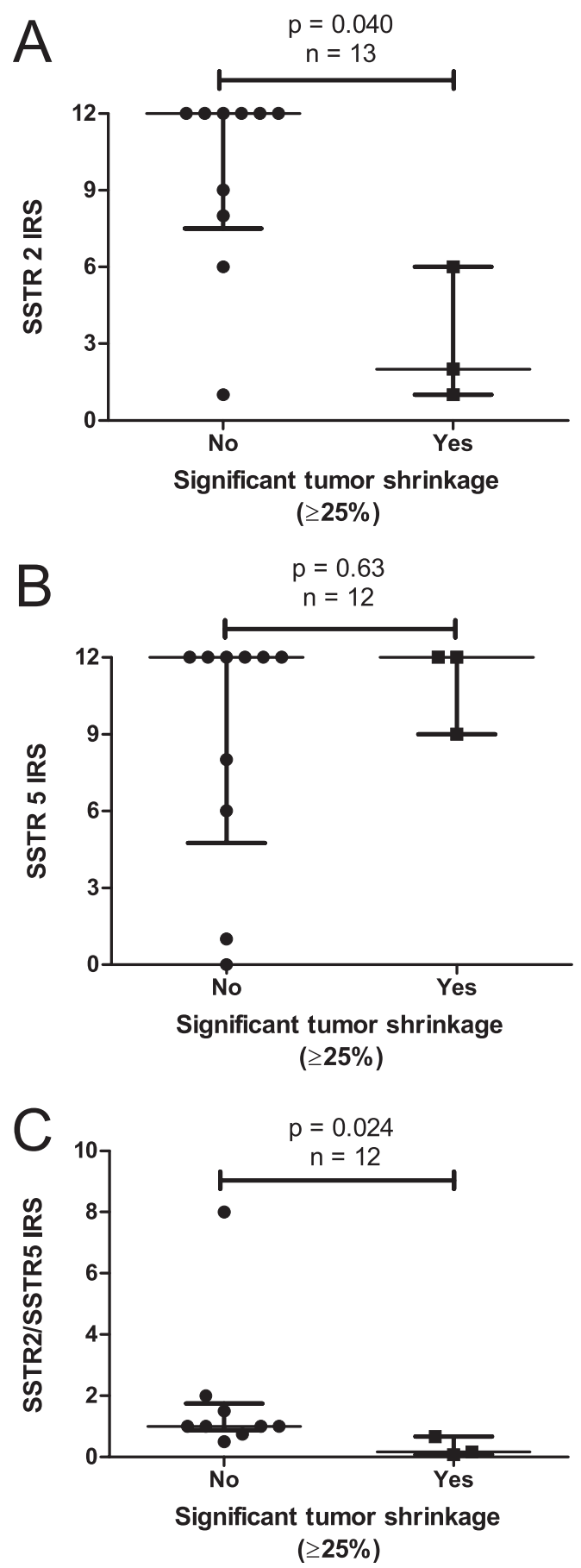

Figure 5

Relation of significant tumor shrinkage and expression of SST receptors. Data are presented as scatter dot plots depicting the median (IQR). $P$ values are for the comparisons between groups and are derived from the Mann-Whitney $U$ test. (A) Patients with significant tumor shrinkage had lower $\mathrm{SST}_{2}$ receptor expression (C) as well as a lower $\mathrm{SST}_{2} / \mathrm{SST}_{5}$ receptor ratio, (B) while $\mathrm{SST}_{5}$ receptor expression was not significantly different between the groups. SSTR, somatostatin receptor. 
Table 2 Determinants of IGF-1 (x ULN) during 3 months of PAS-LAR.

\begin{tabular}{|c|c|c|}
\hline Characteristic & Multivariable Beta $(95 \% \mathrm{Cl})$ & $P$ value \\
\hline Gender (female) & $-0.06(-0.36-0.23)$ & 0.66 \\
\hline T2-signal adenoma/pituitary or GM (baseline) & $-0.29(-0.56$ to -0.01$)$ & 0.045 \\
\hline Significant tumor shrinkage $(\geq 25 \%)$ & $0.34(0.02-0.65)$ & 0.035 \\
\hline
\end{tabular}

GM, gray matter; IGF-1, insulin-like growth factor 1; PAS-LAR, pasireotide long-acting release; ULN, upper limit of normal.

\section{Immunohistochemistry of the adenoma}

We observed that patients with significant tumor shrinkage had lower $\mathrm{SST}_{2}$ receptor (median (IQR): 2.0 (1.0-6.0) vs 12.0 (7.5-12.0), $P=0.040$; Fig. $5 \mathrm{~A}$ ) as well as lower $\mathrm{SST}_{2} / \mathrm{SST}_{5}$ receptor ratio expression (median (IQR): $0.2(0.1-0.7)$ vs $1.0(0.9-1.8), P=0.024$; Fig. 5 C). SST $_{5}$ receptor expression did not differ significantly between the groups (median (IQR): $12.0 \quad(9.0-12.0)$ vs 12.0 (4.8-12.0), $P=0.63$; Fig. 5B).

From the 13 tissue samples included for IHC analysis, two tissue samples (14\%) were derived from patients with increased T2-signal intensity. We did not observe a significant difference in $\mathrm{SST}_{2}, \mathrm{SST}_{5}$ receptor expression, and $\mathrm{SST}_{2} / \mathrm{SST}_{5}$ receptor ratio in patients with increased T2-signal intensity, compared to the total cohort (Table 1).

\section{Hormonal response}

Table 2 shows the regression coefficients and 95\% CIs from the multivariable linear regression model for IGF-1 (x ULN) levels during 3 months of PAS-LAR. A significant inverse association was seen between T2-signal intensity of adenomas at baseline and IGF-1 (x ULN) levels during 3 months of PAS-LAR ( $\beta$ : -0.29 , 95\% CI: -0.56 to -0.01 , $P=0.045$; Table 2). Moreover, we detected a positive association between significant tumor shrinkage and IGF-1 (x ULN) levels during 3 months of PAS-LAR ( $\beta$ : 0.34, 95\% CI: 0.02-0.65, $P=0.035$; Table 2). In other words, higher T2-signal intensity adenomas at baseline and less tumor shrinkage were associated with lower IGF-1 (x ULN) levels during 3 months of PAS-LAR.

\section{Discussion}

Our study provides the novel finding that in acromegaly patients the tumoral responsiveness to PAS-LAR treatment is directly correlated to hormonal unresponsiveness to both first-generation SRL and PAS-LAR treatment and a lower $\mathrm{SST}_{2}$ receptor expression. A higher baseline T2-signal intensity was associated with a better biochemical response to PAS-LAR after 3 months. Interestingly, as previously shown in our cohort, we are the first to observe a substantial increase in T2-signal intensity of the adenoma in response to PAS-LAR (26). Increased T2-signal intensity indicates cystic degeneration, tumor cell necrosis, or both, suggesting an anti-tumor effect of PAS-LAR. This increase was particularly substantial (>50\%) in eight patients.

This potential antitumor activity of PAS-LAR emerged when we observed a change in mean T2-signal intensity ratio in somatotroph adenomas after PAS-LAR treatment in our cohort. In contrast, we did not observe any significant changes in the mean T2-signal intensity ratio after first-generation SRL treatment (14). Compared to the total cohort, patients with increased T2-signal intensity in whom (biochemical) disease activity could not be controlled by SRLs, greater reduction of IGF-1 levels was observed during 3 months of PAS-LAR treatment. These patients had larger adenomas but did not present more often with significant tumor shrinkage. However, we consider it necessary to differentiate tumor shrinkage from cell degeneration, tumor necrosis, or both (e.g. antitumor effects). While PAS-LAR shows similar inhibitory effects on tumor growth as octreotide in GH secreting adenoma cell cultures (18), it additionally showed antitumor effects (cell degeneration or tumor necrosis) in a subgroup of patients, which have not been observed with first-generation SRLs. Long-term data collection is required to further understand the anti-tumor effects of PAS-LAR. Among the SST receptor subtypes, only $\mathrm{SST}_{2}$ and $\mathrm{SST}_{3}$ might be responsible for the PAS-LAR-induced cystic degeneration, tumor cell necrosis, or both, and may reduce disease activity and might result in longterm remission. Studying the effects of PAS-LAR on pituitary histology might help unravel the differences in SST receptors specific signaling pathways and should be further explored.

Although previous clinical studies $(5,6)$ and our study suggest that PAS-LAR might exert a greater effect on tumor control, especially in patients whose disease is inadequately controlled on first-generation SRLs, we observed a significant tumor volume increase in six 
patients. The magnitude of the observed tumor volume increase is similar to previous studies $(5,6,27)$. This observation was not clinically relevant in most $(5 / 6$, $83.3 \%$ ) patients, of which three harbored microadenomas. We considered tumor growth to be clinically relevant only in those with emerging growth that affects the treatment approach (e.g. surgical intervention and/or stereotactic radiosurgery) within 2 years of study entry. However, one of the six patients experienced a substantial ( $>50 \%)$ increase in tumor size during 9 months of PAS-LAR treatment (26 $270 \mathrm{~mm}^{3}$ vs $12495 \mathrm{~mm}^{3}$ ) and underwent surgery. This patient was diagnosed during adolescence and investigations for germline mutations in the aryl hydrocarbon receptor-interacting protein (AIP) and multiple endocrine neoplasia type 1 (MEN1) genes detected no abnormalities. It is noteworthy that this patient showed aggressive and treatment-resistant pituitary tumor behavior throughout previous treatment modalities and exhibited patient and tumor characteristics predictive of aggressive tumor behavior in the future (e.g. young age at diagnosis, large tumor size, and high GH secretion) (28). These young patients with large tumors and/or those with high pretreatment levels of GH particularly warrant close monitoring for continued tumor progression, regardless of treatment modality.

Hormone responsiveness (i.e. lower IGF-1 levels) during PAS-LAR treatment in the total cohort was associated with higher T2-signal intensity. This finding is contrary to previous studies on first-generation SRLs, reporting greater IGF-1 reduction during SRL treatment in T2-hypointense adenomas $(9,11,13,14)$. Thus, T2-weighted MRI signal intensity may provide complementary predictive information in relation to the responses to both kinds of drugs. However, these results are not unexpected considering that patients with higher T2-signal intensity adenomas may have lower IGF-1 levels at diagnosis (10). Consequently, these patients require less IGF-1 reduction in order to obtain biochemical control.

Most studies have reported hormone secretion and cell growth to be synchronous, as tumors responding to first-generation somatostatin analogs with GH inhibition also exhibit tumor shrinkage. However, we observed a dissociation between achieved IGF-1 levels and the presence of significant tumor shrinkage during PASLAR treatment. Two mechanisms may account for this dissociation.

First, the dissociation between anti-secretory and anti-proliferative effects can be based on the different roles of SST receptor subtypes in GH secreting tumors. To our knowledge, this is the first reported incidence of an additional (significant) decrease in tumor volume after switching from first-generation SRL to PAS-LAR treatment. Our results give a clear indication that this dissociation can be based on the different roles of SST receptor subtypes. Previous in vitro studies $(19,29,30)$ and our data (23), as previously reported by Muhammad et al., indicate that, overall, pasireotide exerts its anti-secretory activity mainly by the activation of $\mathrm{SST}_{2}$. Patients with significant tumor shrinkage had significantly lower $\mathrm{SST}_{2}$ receptor expression, as well as a lower $\mathrm{SST}_{2} / \mathrm{SST}_{5}$ ratio compared to patients without significant tumor shrinkage, which probably accounts for the lack of biochemical control. Yet, we have to point out that we analyzed the tissue samples from only 13 patients. Although there is no evidence that $\mathrm{SST}_{5}$ receptor expression is affected by presurgical first-generation SRL treatment, it has been widely demonstrated that patients receiving SRL treatment prior to surgery show significantly lower $\mathrm{SST}_{2}$ receptor protein expression compared to medically naive patients $(15,16,19,31)$. Even though this is not evident in our cohort, a pooled analysis of SRL-pretreated and medically naive patients can introduce bias. Exclusion of the SRLpretreated patients $(n=4)$ from our analysis did not affect our observation, since the difference in $\mathrm{SST}_{2}$ receptor expression between patients with or without significant tumor shrinkage remained significant.

Secondly, the tumor size may explain why significant tumor shrinkage is not accompanied by lower IGF-1 levels. In our cohort, absolute tumor shrinkage during PAS-LAR treatment was positively correlated with larger tumors and higher GH levels at baseline. These results confirm and build upon a previous study stating that IGF-1 levels remain unaffected above a certain threshold of GH concentrations (32). Knowing that larger tumors secrete more $\mathrm{GH}$, it becomes apparent that PAS-LAR can affect tumor size and thereby GH levels, without affecting IGF-1 levels. In fact, we observed significantly decreased GH levels during PAS-LAR therapy in subjects from the PAPE study as previously reported by Muhammad et al. $(7,8)$, which can be explained by the suppressive effect of PAS-LAR on GH secretion. This finding is also compatible with reports of increased GH levels during PEGV treatment (33) and the lower GH levels can be explained by the discontinuation of or further dose reduction in PEGV.

A strength of our study lies in the relatively large number of patients in which the hormone and tumor response to different treatment options were systematically investigated, including data on T2-signal intensity and SST receptor expression of somatotroph adenomas. The main limitations of our study lie in the 
retrospective collection of data on IGF-1 levels during first-generation SRL treatment and the use of different IGF-1 assays during SRL treatment. Another limitation of our study is the lack of generalizability, as most selected patients did not receive surgery and may not reflect the general population of acromegaly patients. However, the fact that our patients had a high degree of resistance to first-generation SRL therapy fits with the second-line indication of PAS-LAR in most non-US countries. At last, we should mention that most previous studies expressed IGF-1 differently to evaluate efficacy; with most studies using the achieved reduction in IGF-1 level at any point $(9,10,11,34)$, while we used IGF-1 levels expressed as ULN during 3 and 9 months in our study.

In conclusion, patients showing no biochemical response to first- or second-generation somatostatin analogs with particularly large tumors with a lower $\mathrm{SST}_{2}$ receptor expression are prone to achieve tumor shrinkage during PAS-LAR treatment. On the contrary, lower IGF-1 levels during PAS-LAR are associated with a higher $\mathrm{SST}_{2}$ receptor expression and a higher T2-signal intensity. In patients with increased T2-signal intense adenomas, PASLAR-induced cystic degeneration, tumor cell necrosis, or both, might reduce disease activity by achieving greater IGF-1 reduction in patients with relatively larger adenomas. This poses the question whether possibly $\mathrm{SST}_{3}$ or other SST receptors may be relevant as well during PAS-LAR treatment in mediating tumor shrinkage or inducing cell degeneration, tumor cell necrosis, or both. Future research should aim at elucidating differences in the hormone and tumor responsiveness in response to PAS-LAR treatment. To predict which subgroup of patients will benefit from treatment with this multireceptor ligand and the consequences for the clinical management of acromegaly remains to be elucidated.

\section{Declaration of interest}

E C Coopmans, J J Schneiders, N El-Sayed, N S Erler, J Potorac, and A Muhammad have nothing to disclose. $\mathrm{L} J$ Hofland received investigatorinitiated research grants from Novartis and Ipsen. P Petrossians received speakers and consulting fees from Novartis Pharma, Ipsen Pharma, and Pfizer. A J van der Lely is a consultant to Pfizer Inc. and Millendo Therapeutics and received grants from Pfizer Inc. S J C M M Neggers received research grants and speaker fees from Ipsen Pharma, Novartis Pharma, and Pfizer Inc. and consulting fees from Pfizer, Crinetics, and Ipsen Pharma.

\section{Funding}

The study did not receive any specific grant from any funding agency in the public, commercial, or non-profit sector.

\section{Acknowledgements}

The authors acknowledge the clinical chemist (Sjoerd A A van den Berg) and neurosurgeons (Alof H G Dallenga and Ian K Haitsma). All contributed to the study.

\section{References}

1 Melmed S. Acromegaly pathogenesis and treatment. Journal of Clinical Investigation 2009119 3189-3202. (https://doi.org/10.1172/ JCI39375)

2 Melmed S, Bronstein MD, Chanson P, Klibanski A, Casanueva FF, Wass JAH, Strasburger CJ, Luger A, Clemmons DR \& Giustina A. A Consensus Statement on acromegaly therapeutic outcomes. Nature Reviews: Endocrinology 201814 552-561. (https://doi.org/10.1038/ s41574-018-0058-5)

3 Signifor LAR (package insert). East Hanover: Novartis Pharmaceuticals Corporation, 2014.

4 Signifor LAR (summary of product characteristics). Basel: Novartis Pharma AG, 2014.

5 Colao A, Bronstein MD, Freda P, Gu F, Shen CC, Gadelha M, Fleseriu M, van der Lely AJ, Farrall AJ, Hermosillo Resendiz K et al. Pasireotide versus octreotide in acromegaly: a head-to-head superiority study. Journal of Clinical Endocrinology and Metabolism 201499 791-799. (https://doi.org/10.1210/jc.2013-2480)

6 Gadelha MR, Bronstein MD, Brue T, Coculescu M, Fleseriu M, Guitelman M, Pronin V, Raverot G, Shimon I, Lievre KK et al. Pasireotide versus continued treatment with octreotide or lanreotide in patients with inadequately controlled acromegaly (PAOLA): a randomised, phase 3 trial. Lancet: Diabetes and Endocrinology 20142 875-884. (https://doi.org/10.1016/S2213-8587(14)70169-X)

7 Muhammad A, van der Lely AJ, Delhanty PJD, Dallenga AHG, Haitsma IK, Janssen J \& Neggers S. Efficacy and safety of switching to pasireotide in acromegaly patients controlled with pegvisomant and first-generation somatostatin analogues (PAPE study). Journal of Clinical Endocrinology and Metabolism 2017103 586-595. (https://doi. org/10.1210/jc.2017-02017)

8 Muhammad A, Coopmans EC, Delhanty PJD, Dallenga AHG, Haitsma IK, Janssen JAMJL, Van der Lely AJ \& Neggers SJCMM. Efficacy and safety of switching to pasireotide in acromegaly patients controlled with pegvisomant and somatostatin analogues: PAPE extension study. European Journal of Endocrinology 2018179 269-277. (https://doi.org/10.1530/EJE-18-0353)

9 Potorac I, Petrossians P, Daly AF, Alexopoulou O, Borot S, SahnounFathallah M, Castinetti F, Devuyst F, Jaffrain-Rea ML, Briet C et al. T2-weighted MRI signal predicts hormone and tumor responses to somatostatin analogs in acromegaly. Endocrine-Related Cancer 2016 23 871-881. (https://doi.org/10.1530/ERC-16-0356)

10 Potorac I, Petrossians P, Daly AF, Schillo F, Ben Slama C, Nagi S, Sahnoun M, Brue T, Girard N, Chanson P et al. Pituitary MRI characteristics in 297 acromegaly patients based on T2-weighted sequences. Endocrine-Related Cancer 201522 169-177. (https://doi. org/10.1530/ERC-14-0305)

11 Heck A, Ringstad G, Fougner SL, Casar-Borota O, Nome T, RammPettersen J \& Bollerslev J. Intensity of pituitary adenoma on T2-weighted magnetic resonance imaging predicts the response to octreotide treatment in newly diagnosed acromegaly. Clinical Endocrinology 201277 72-78. (https://doi.org/10.1111/j.13652265.2011.04286.x)

12 Hagiwara A, Inoue Y, Wakasa K, Haba T, Tashiro T \& Miyamoto T. Comparison of growth hormone-producing and non-growth hormone-producing pituitary adenomas: imaging characteristics and pathologic correlation. Radiology 2003228 533-538. (https://doi. org/10.1148/radiol.2282020695) 
13 Shen M, Zhang Q, Liu W, Wang M, Zhu J, Ma Z, He W, Li S, Shou X, Li Y et al. Predictive value of T2 relative signal intensity for response to somatostatin analogs in newly diagnosed acromegaly. Neuroradiology 201658 1057-1065. (https://doi.org/10.1007/s00234016-1728-4)

14 Bonneville F, Riviere LD, Petersenn S, Bevan J, Houchard A, Sert C, Caron PJ \& PRIMARYS Study group. MRI T2 signal intensity and tumor response in patients with GH-secreting pituitary macroadenoma: PRIMARYS post-hoc analysis. European Journal of Endocrinology 2019180 155-164. (https://doi.org/10.1530/EJE-180254)

15 Gatto F, Feelders RA, van der Pas R, Kros JM, Waaijers M, SprijMooij D, Neggers SJ, van der Lelij AJ, Minuto F, Lamberts SW et al. Immunoreactivity score using an anti-sst2A receptor monoclonal antibody strongly predicts the biochemical response to adjuvant treatment with somatostatin analogs in acromegaly. Journal of Clinical Endocrinology and Metabolism 201398 E66-E71. (https://doi. org/10.1210/jc.2012-2609)

16 Casar-Borota O, Heck A, Schulz S, Nesland JM, Ramm-Pettersen J, Lekva T, Alafuzoff I \& Bollerslev J. Expression of SSTR2a, but not of SSTRs 1,3 , or 5 in somatotroph adenomas assessed by monoclonal antibodies was reduced by octreotide and correlated with the acute and long-term effects of octreotide. Journal of Clinical Endocrinology and Metabolism 201398 E1730-E1739. (https://doi.org/10.1210/ jc.2013-2145)

17 Schmid HA. Pasireotide (SOM230): development, mechanism of action and potential applications. Molecular and Cellular Endocrinology 2008286 69-74. (https://doi.org/10.1016/j.mce.2007.09.006)

18 Ibanez-Costa A, Rivero-Cortes E, Vazquez-Borrego MC, Gahete MD, Jimenez-Reina L, Venegas-Moreno E, de la Riva A, Arraez MÁ, Gonzalez-Molero I, Schmid HA et al. Octreotide and pasireotide (dis) similarly inhibit pituitary tumor cells in vitro. Journal of Endocrinology 2016231 135-145. (https://doi.org/10.1530/JOE-16-0332)

19 Gatto F, Feelders RA, Franck SE, van Koetsveld PM, Dogan F, Kros JM, Neggers SJCMM, van der Lely AJ, Lamberts SWJ, Ferone D et al. In vitro head-to-head comparison Between octreotide and pasireotide in GH-secreting pituitary adenomas. Journal of Clinical Endocrinology and Metabolism 2017102 2009-2018. (https://doi.org/10.1210/jc.201700135)

20 Manolopoulou J, Alami Y, Petersenn S, Schopohl J, Wu Z, Strasburger CJ \& Bidlingmaier M. Automated 22-kD growth hormone-specific assay without interference from pegvisomant. Clinical Chemistry 201258 1446-1456. (https://doi.org/10.1373/ clinchem.2012.188128)

21 Bidlingmaier M, Friedrich N, Emeny RT, Spranger J, Wolthers OD, Roswall J, Korner A, Obermayer-Pietsch B, Hubener C, Dahlgren J et al. Reference intervals for insulin-like growth factor-1 (IGF-I) from birth to senescence: results from a multicenter study using a new automated chemiluminescence IGF-I immunoassay conforming to recent international recommendations. Journal of Clinical Endocrinology and Metabolism 201499 1712-1721. (https://doi. org/10.1210/jc.2013-3059)

22 Lundin P \& Pedersen F. Volume of pituitary macroadenomas: assessment by MRI. Journal of Computer Assisted Tomography $1992 \mathbf{1 6}$ 519-528. (https://doi.org/10.1097/00004728-199207000-00004)

23 Muhammad A, Coopmans EC, Gatto F, Franck SE, Janssen JAMJL, van der Lely AJ, Hofland LJ \& Neggers SJCMM. Pasireotide responsiveness in acromegaly is mainly driven by somatostatin receptor subtype 2 expression. Journal of Clinical Endocrinology and Metabolism 2019104 915-924. (https://doi.org/10.1210/jc.201801524)

24 Remmele W \& Stegner HE. Recommendation for uniform definition of an immunoreactive score (IRS) for immunohistochemical estrogen receptor detection (ER-ICA) in breast cancer tissue. Der Pathologe 19878 138-140.

25 Neggers SJ, Franck SE, de Rooij FW, Dallenga AH, Poublon RM, Feelders RA, Janssen JA, Buchfelder M, Hofland LJ, Jorgensen JO et al. Long-term efficacy and safety of pegvisomant in combination with long-acting somatostatin analogs in acromegaly. Journal of Clinical Endocrinology and Metabolism 201499 3644-3652. (https://doi. org/10.1210/jc.2014-2032)

26 Coopmans EC, van der Lely AJ, Schneiders JJ \& Neggers SJCMM. Potential antitumour activity of pasireotide on pituitary tumours in acromegaly. Lancet: Diabetes and Endocrinology $20197425-426$. (https://doi.org/10.1016/S2213-8587(19)30113-5)

27 Bronstein MD, Fleseriu M, Neggers S, Colao A, Sheppard M, Gu F, Shen CC, Gadelha M, Farrall AJ, Hermosillo Resendiz K et al. Switching patients with acromegaly from octreotide to pasireotide improves biochemical control: crossover extension to a randomized, double-blind, phase III study. BMC Endocrine Disorders 20161616. (https://doi.org/10.1186/s12902-016-0096-8)

28 Besser GM, Burman P \& Daly AF. Predictors and rates of treatment-resistant tumor growth in acromegaly. European Journal of Endocrinology 2005153 187-193. (https://doi.org/10.1530/ eje.1.01968)

29 Hofland LJ, van der Hoek J, van Koetsveld PM, de Herder WW, Waaijers M, Sprij-Mooij D, Bruns C, Weckbecker G, Feelders R, van der Lely AJ et al. The novel somatostatin analog SOM230 is a potent inhibitor of hormone release by growth hormone- and prolactinsecreting pituitary adenomas in vitro. Journal of Clinical Endocrinology and Metabolism 200489 1577-1585. (https://doi.org/10.1210/ jc.2003-031344)

30 Jaquet P, Saveanu A, Gunz G, Fina F, Zamora AJ, Grino M, Culler MD, Moreau JP, Enjalbert A \& Ouafik LH. Human somatostatin receptor subtypes in acromegaly: distinct patterns of messenger ribonucleic acid expression and hormone suppression identify different tumoral phenotypes. Journal of Clinical Endocrinology and Metabolism 200085 781-792. (https://doi.org/10.1210/jcem.85.2.6338)

31 Plockinger U, Albrecht S, Mawrin C, Saeger W, Buchfelder M, Petersenn S \& Schulz S. Selective loss of somatostatin receptor 2 in octreotide-resistant growth hormone-secreting adenomas. Journal of Clinical Endocrinology and Metabolism 200893 1203-1210. (https:// doi.org/10.1210/jc.2007-1986)

32 Barkan AL, Beitins IZ \& Kelch RP. Plasma insulin-like growth factor-I/ somatomedin-C in acromegaly: correlation with the degree of growth hormone hypersecretion. Journal of Clinical Endocrinology and Metabolism 198867 69-73. (https://doi.org/10.1210/jcem-67-1-69)

33 Trainer PJ, Drake WM, Katznelson L, Freda PU, Herman-Bonert V, van der Lely AJ, Dimaraki EV, Stewart PM, Friend KE, Vance ML et al. Treatment of acromegaly with the growth hormone-receptor antagonist pegvisomant. New England Journal of Medicine 2000342 1171-1177. (https://doi.org/10.1056/NEJM200004203421604)

34 Heck A, Emblem KE, Casar-Borota O, Ringstad G \& Bollerslev J. MRI $\mathrm{T} 2$ characteristics in somatotroph adenomas following somatostatin analog treatment in acromegaly. Endocrine 201653 327-330. (https:// doi.org/10.1007/s12020-015-0816-2)

Received 18 October 2019

Revised version received 20 March 2020

Accepted 9 April 2020 